\title{
Elucidation of the Microstructure of Carbon-Carbon Composites by Raman Microscopy
}

\author{
Fran Adar, Ph.D.*, Eunah Lee, Ph.D.*, Sergey Mamedov, Ph.D.*, and Andrew Whitley, Ph.D.* \\ *Horiba Scientific, 3880 Park Ave., Edison, NJ 08820
}

Carbon-carbon composites are used in aerospace materials as well as some automotive, high-end sports and helmet applications. Their advantages include stiffness, strength, and light weight, all properties which are superior to those of steel or other metals, and they can be used at high temperatures. However, even though they are less brittle than conventional ceramics, they do lack impact resistance. Because of the costs involved in manufacturing these materials, and the importance of their applications, especially in aerospace, any technique that can characterize them is of interest. Images of a composite created on a Raman microscope has been used to characterize a carbon-carbon composite. It is shown the information is encoded in both details of the spectral features and polarization behavior. This information can be useful in engineering these materials.

In these materials carbon fibers are woven into cloth and shaped into a "preform" which is then fused into a solid by one or more of a variety of methods that provide a matrix of carbon surrounding the fibers. These methods include impregnation and pyrolysis of organic resins, and chemical vapor deposition to fill the voids created during the pyrolysis. They can be used at high temperatures, where thermal shock resistance and/or a low thermal expansion coefficient are required. Consequently they maintain their mechanical properties to temperatures above $2000^{\circ} \mathrm{C}$. For applications in missiles and the space shuttle, the surfaces have been converted to $\mathrm{SiC}$ in order to prevent atmospheric oxidation; $\mathrm{SiC}$ can also be characterized by Raman microscopy, but will not be addressed in this work.

The material properties of these composites depend on the fiber fraction, the fiber type, the textile weave, the properties of the fibers and the properties of the matrix material. The properties of the fibers themselves depend on the precursor material, the method of production, the degree of orientation, and the degree of graphitization, which in turn, will depend on tensioning during fiber formation. Clearly the effectiveness of the matrix material will depend on precursor and manufacturing method, densification, and adhesion. Because of the high cost of production, carboncarbon composite use is limited.

In order to assess whether Raman can be helpful in characterizing carbon-carbon composites, polarized maps were acquired. A polarized micrograph of a carbon-carbon composite that had been cross-sectioned and polished in a plane perpendicular to the fiber direction is shown in Figure 1. In crossed polars the fibers are grey, while the spherulites are a mixture of bright white and black. It is well known that spherulites with mixed orientation will exhibit contrast in polarized light microscopy. The question is whether any further information can be provided by Raman microscopy.

Graphite is composed of stacks of planes of $\mathrm{sp}^{2}$-bonded carbon with the layers staggered. There are 2 planes in the unit cell, and the symmetry is $\mathrm{D}_{6 \mathrm{~h}}^{4}$. There are two doubly degenerate, Raman-active, in-plane, $E_{2 \mathrm{~g}}$ modes, with frequencies of 1582 and $42 \mathrm{~cm}^{-1}$, the former being known as the $\mathrm{G}$ mode. 
The lower frequency mode corresponds to shear motion of the two planes, and can only be observed in systems capable of recording frequencies well below $100 \mathrm{~cm}^{-1}$. A continuum of structures of $\mathrm{sp}^{2}$ bonded carbon has been characterized by Raman spectroscopy. If one starts with graphite, and grinds it into smaller crystallites, a 'disorder' or D band appears [1] somewhere in the vicinity of 1280 and $1400 \mathrm{~cm}^{-1}$, depending on the excitation wavelength [2]. This band was originally assigned to scattering from the normally forbidden edge of the Brillouin Zone, but allowed because of the limited size of the crystallites. In disordered materials there is also a band at about $1620 \mathrm{~cm}^{-1}$ which is called the D' band. A careful analysis of many observations, including the inequivalence of the Stokes and anti-Stokes frequencies, indicates that the D band appears because of symmetry lowering and there are 'double resonances' with electronic transitions [3].

XY maps were recorded from a carbon composite under parallel and perpendicular polarization conditions. Figure 1 shows the optical micrograph in polarized light of the mapped composite, and the results of the Raman measurements, using model spectra to create the Raman images. Figure 2 shows the model spectra derived from the hyperspectral cube. Under perpendicular polarization conditions, one less factor was required to account for the variance. The presentation will include a discussion of what these observations can imply about the physical state of the composites.
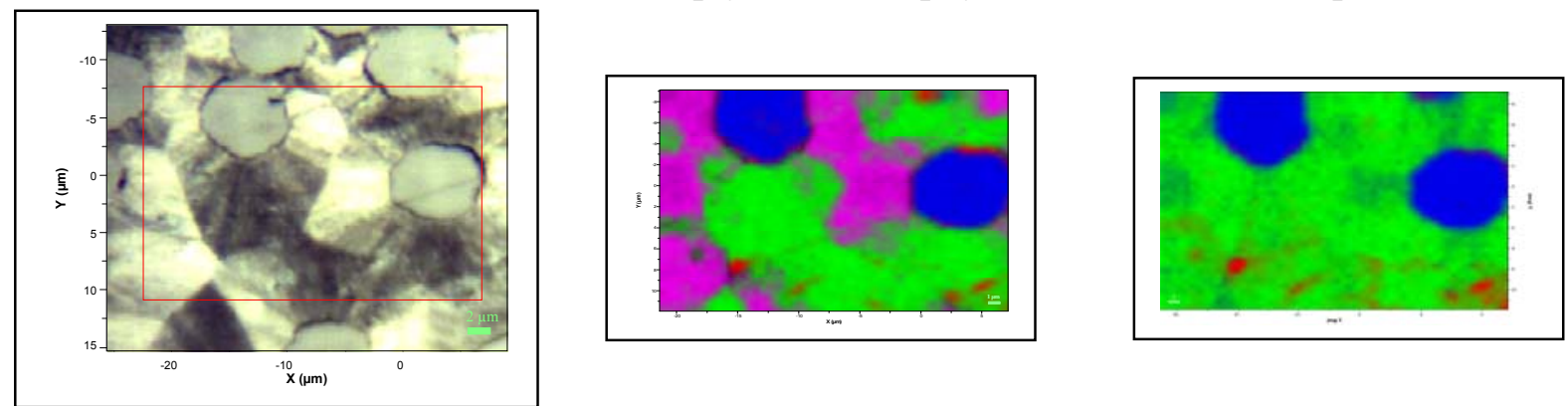

Figure 1. Polarized light micrograph (left) and polarized Raman maps using parallel (middle) and perpendicular (right) analyzers.
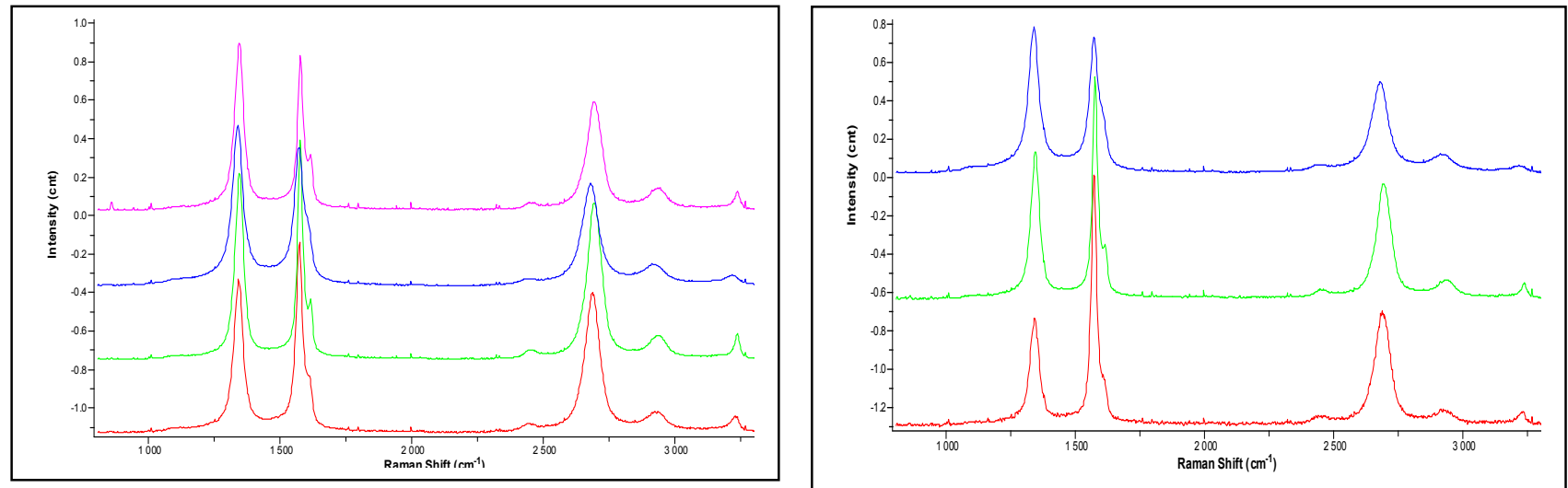

Figure 2. Model spectra calculated from XY maps for paralleI and perpendicular polarization conditions.

\footnotetext{
${ }^{1}$ F. Tuinstra and J.L. Koenig, Raman Spectrum of Graphite, J. Chem. Phys. 1970 53m 1126

${ }^{2}$ Y. Wang, D.C. Alsmeyer and R.L. McCreery, Raman Spectroscopy of Carbon Materials: Structural Basis of Observed Spectra Chem Mater. 1990, 2, 557-563

${ }^{3}$ S. Reich and C. Thomsen, Raman Spectroscopy of Graphite, originally published on-line by the Royal Society 14 Sept. 2004 (10.1098/rsta.2004.1454) Phil. Trans. R. Soc. Lond. A (2004) 2271-2288
} 